\title{
Numerical Solution of an Exterior Neumann Problem Using a Double Layer Potential
}

\author{
By J. Giroire and J. C. Nedelec
}

Abstract. We give here a variational formulation in $H^{1 / 2}(\Gamma) / \mathrm{R}$ of the exterior Neumann problem for the Laplace operator using a double layer potential. This formulation is then applied to the construction of a finite element method. Optimal error estimates are given.

Introduction. Solving boundary value problems for partial differential operators by integral equation methods is not a new idea. However, the classical way to do it consists in representing the unknown solution as a potential of the type that will lead to an integral equation of the second kind. Then, Fredholm's theorems can be used. Thus, the Dirichlet problem is usually solved with the help of a double layer potential, and the Neumann problem with the use of a single layer potential.

We shall have a different point of view. Our aim will be to obtain a variational formulation of the problem in order to obtain the existence and unicity of a solution and error estimates. This philosophy leads to opposite choices for the representation of the solution. Thus, J. C. Nedelec and J. Planchard, for the three-dimensional case, and M. N. Leroux for the two-dimensional case, have solved the Dirichlet problem by using a single layer potential. We propose here the solution of a Neumann problem by using a double layer potential.

Let $\Omega$ be a bounded open set of $\mathbf{R}^{3}$. Let $\Gamma$ be the boundary of $\Omega$ and $\Omega^{c}$ denote the complementary set of $\bar{\Omega}$.

We assume that $\Gamma$ is sufficiently smooth, and we put the coordinates' origin in

$\Omega$. We shall write

$\vec{n}$, for the exterior normal to $\Gamma$,

$r$, for the distance to the origin,

$[v]=\left.v\right|_{\Gamma} ^{\text {int }}-\left.v\right|_{\Gamma} ^{\text {ext }}$, for the jump through $\Gamma$, of the function $v$ defined in $\mathbf{R}^{3}$.

I. The Exterior Neumann Problem for the Laplace Operator. Let us consider the following problem.

$\left(P_{1}\right)\left\{\begin{array}{l}\text { Find } u_{1} \in W_{0}^{1}\left(\Omega^{c}\right)=\left\{v \in D^{\prime}\left(\Omega^{c}\right) \mid v / r \in L^{2}\left(\Omega^{c}\right), D v \in L^{2}\left(\Omega^{c}\right)\right\}, \text { such that } \\ \Delta u_{1}=0 \text { in } \Omega^{c}, \\ \partial u_{1} / \partial n=g_{1} \in H^{-1 / 2}(\Gamma) .\end{array}\right.$

We have the

Received September 12, 1977.

AMS (MOS) subject classifications (1970). Primary $65 \mathrm{R} 05$.

Copyright $\odot 1978$, American Mathematical Society 
Proposition I-1. Problem $\left(P_{1}\right)$ has one and only one solution.

Proof. It is a straightforward consequence of the fact that $\|\operatorname{grad} v\|_{L^{2}\left(\Omega^{c}\right)}$ is a norm on $W_{0}^{1}\left(\Omega^{c}\right)$ equivalent to the definition norm [4, Theorem II-2, p. 20].

For $g_{1}$ arbitrary in $H^{-1 / 2}(\Gamma)$, it is impossible to find a harmonic extension of $u_{1}$ in $\Omega$. Let then $\lambda$ and $u_{0}$ be defined by

$$
\lambda=\left\langle g_{1}, 1\right\rangle_{H^{-1 / 2}(\Gamma) \times H^{1 / 2}(\Gamma)} \text { and } \quad u_{0}(x)=\frac{\lambda}{4 \pi} \frac{1}{\left|x-x_{0}\right|},
$$

where $x_{0}$ is an arbitrary point of $\Omega$. Let us take

$$
u=u_{1}-u_{0} \text { and } g=g_{1}-\frac{\partial u_{0}}{\partial n}
$$

Then, $u$ is harmonic in $\Omega^{c}$ and can be harmonically extended in $\Omega$. We are thus led to problem $(P)$.

$$
\left\{\begin{array}{l}
\text { Find } u \in\left(H^{1}(\Omega) / \mathbf{R}\right) \times W_{0}^{1}\left(\Omega^{c}\right) \text { such that } \\
\Delta u=0 \text { in } \Omega \text { and } \Omega^{c} \\
\partial u / \partial n=g \in H_{0}^{-1 / 2}(\Gamma)=\left\{h \in H^{-1 / 2}(\Gamma) \mid\langle h, 1\rangle=0\right\}
\end{array}\right.
$$

We have then

Theorem I-1. Problem (P) has one and only one solution.

Proof. We have only to split up Problem $(P)$ into an interior problem and an exterior one. For an interior problem, the result is well known [9]. For an exterior problem, it is the result stated in Proposition I-1.

In order to introduce the formulation on $\Gamma$ which we are interested in, we shall need a problem $\left(P^{\prime}\right)$ that we are going to define now. First, let us define

$$
\begin{aligned}
H^{1}(\Delta ; \Omega) & =\left\{v \in H^{1}(\Omega), \Delta v \in L^{2}(\Omega)\right\}, \\
W^{1}\left(\Delta ; \Omega^{c}\right) & =\left\{v \in W_{0}^{1}\left(\Omega^{c}\right), r \Delta v \in L^{2}\left(\Omega^{c}\right)\right\}, \\
K & =\left\{v \in\left(H^{1}(\Delta ; \Omega) / \mathbf{R}\right) \times W^{1}\left(\Delta ; \Omega^{c}\right) \mid \operatorname{supp}(\Delta v) \subset \Gamma,[\partial v / \partial n]=0\right\}, \\
\|v\|_{K} & =\left(\int_{\Omega}|\operatorname{grad} v|^{2} d x+\int_{\Omega}^{c}|\operatorname{grad} v|^{2} d x\right)^{1 / 2}
\end{aligned}
$$

Problem $\left(P^{\prime}\right)$ is the following:

$$
\text { Find } u \in K, \quad \text { such that }[u]=q \in H^{1 / 2}(\Gamma) / \mathbf{R} \text {. }
$$

We have then

Proposition I-2. Problem ( $P^{\prime}$ ) has one and only one solution.

Proof. For $u \in H^{1}(\Delta ; \Omega)$ we have the following Green's formula [9].

$\forall v \in H^{1}(\Omega), \quad \int_{\Omega} \operatorname{grad} u \operatorname{grad} v d x=-\int_{\Omega} \Delta u v d x+\left\langle\frac{\partial u}{\partial n}, v\right\rangle_{H^{-1 / 2}(\Gamma) \times H^{1 / 2}(\Gamma)}$.

In the same way, one can prove for $u \in W^{1}\left(\Delta ; \Omega^{c}\right)$ the following Green's formula [4]. 
$\forall v \in W_{0}^{1}\left(\Omega^{c}\right), \quad \int_{\Omega^{c}} \operatorname{grad} u \operatorname{grad} v d x=-\int_{\Omega^{c}} \Delta u v d x-\left\langle\frac{\partial u}{\partial n}, v\right\rangle_{H^{-1 / 2}(\Gamma) \times H^{1 / 2}(\Gamma)}$.

Let us now consider $u \in K$. We have

$$
\begin{gathered}
\forall v \in\left(H^{1}(\Omega) / \mathbf{R}\right) \times W_{0}^{1}\left(\Omega^{c}\right), \\
\int_{\Omega \cup \Omega_{c}} \operatorname{grad} u \operatorname{grad} v d x=\left\langle\frac{\partial u}{\partial n},[v]\right\rangle_{H_{0}^{-1 / 2}(\Gamma) \times\left(H^{1 / 2}(\Gamma) / \mathbf{R}\right)}
\end{gathered}
$$

If $v$ belongs to $K$ too, we have

$$
\int_{\Omega \cup \Omega^{c}} \operatorname{grad} u \operatorname{grad} v d x=\left\langle\frac{\partial v}{\partial n},[u]\right\rangle_{H_{0}^{-1 / 2}(\Gamma) \times\left(H^{1 / 2}(\Gamma) / \mathrm{R}\right)},
$$

which gives a variational formulation of Problem $\left(P^{\prime}\right)$ and ends the proof.

Thus, Problem $(P)$ defines an isomorphism $J_{0}$ of $H_{0}^{-1 / 2}(\Gamma)$ onto $K$, and Problem $\left(P^{\prime}\right)$ defines an isomorphism $J_{1}$ of $H^{1 / 2}(\Gamma) / \mathrm{R}$ onto $K$. Therefore, $J=J_{1}^{-1} \circ J_{0}$ is an isomorphism of $H_{0}^{-1 / 2}(\Gamma)$ onto $H^{1 / 2}(\Gamma) / R$. We shall see in a moment that a coercive bilinear form corresponds to this isomorphism.

Let $q$ and $q^{\prime}$ belong to $H^{1 / 2}(\Gamma) / \mathbf{R}$ and define $a\left(q, q^{\prime}\right)=\left\langle q, J^{-1}\left(q^{\prime}\right)\right\rangle$. Then, we have

THEOREM I-2. The bilinear form $a$ is symmetrical and positive definite on $H^{1 / 2}(\Gamma) / \mathbf{R}$.

Proof. Define

$$
u=J_{1}(q), \quad v=J_{0}\left(J^{-1}\left(q^{\prime}\right)\right)
$$

Then,

$$
a\left(q, q^{\prime}\right)=\left\langle[u], \frac{\partial v}{\partial n}\right\rangle=\int_{\Omega \cup \Omega^{c}} \operatorname{grad} u \operatorname{grad} v d x=\left\langle[v], \frac{\partial u}{\partial n}\right\rangle=a\left(q^{\prime}, q\right),
$$

and

$$
a(q, q)=\int_{\Omega \cup \Omega^{c}}|\operatorname{grad} u|^{2} d x \geqslant C\|u\|_{\left(H^{1}(\Omega) / R\right) \times w_{0}^{1}\left(\Omega^{c}\right)}
$$

hence,

$$
a(q, q) \geqslant C\|q\|_{H^{1 / 2}(\Gamma) / \mathrm{R}}^{2}
$$

which ends the proof.

Thus, the jump through $\Gamma$ of the solution $u$ of Problem $(P)$ is the solution of the coercive variational problem.

$$
\left\{\begin{array}{l}
\text { Find } q \in H^{1 / 2}(\Gamma) / \mathbf{R} \text { such that } \\
a\left(q, q^{\prime}\right)=\left\langle g, q^{\prime}\right\rangle_{H_{0}^{-1 / 2}(\Gamma) \times\left(H^{i / 2}(\Gamma) / \mathbf{R}\right)}, \forall q^{\prime} \in H^{1 / 2}(\Gamma) / \mathbf{R} .
\end{array}\right.
$$

In order to use these results, we have to find an explicit expression of $a$. This is what we shall do now. 
Proposition I-3. Let $q$ belong to $D(\Gamma)$. The solution $u$ of Problem $\left(P^{\prime}\right)$ can be expressed by

$$
u(y)=-\frac{1}{4 \pi} \int_{\Gamma} q(x) \frac{\partial}{\partial n_{x}}\left(\frac{1}{|x-y|}\right) d \gamma_{x}
$$

Proof. It is an immediate consequence of well-known facts about double layer potentials [13].

ThEOREM I-3. Let $q$ and $q^{\prime}$ belong to $D(\Gamma)$. Then, the bilinear form a has the following expression

$$
a\left(q, q^{\prime}\right)=\frac{1}{8 \pi} \int_{\Gamma} \int_{\Gamma}(q(x)-q(y))\left(q^{\prime}(x)-q^{\prime}(y)\right) \frac{\partial^{2}}{\partial n_{x} \partial n_{y}}\left(\frac{1}{|x-y|}\right) d \gamma_{x} d \gamma_{y}
$$

Proof.

$$
a\left(q, q^{\prime}\right)=\int_{\Gamma} q(y) \frac{\partial}{\partial n_{y}}\left\{-\frac{1}{4 \pi} \int_{\Gamma} q^{\prime}(x) \frac{\partial}{\partial n_{x}}\left(\frac{1}{|x-y|}\right) d \gamma_{x}\right\} d \gamma_{y}=2 A .
$$

Since $a$ is symmetric, we have

$$
a\left(q, q^{\prime}\right)=A+\int_{\Gamma} q^{\prime}(y) \frac{\partial}{\partial n_{y}}\left\{-\frac{1}{8 \pi} \int_{\Gamma} q(x) \frac{\partial}{\partial n_{x}}\left(\frac{1}{|x-y|}\right) d \gamma_{x}\right\} d \gamma_{y}=A+B
$$

On the other hand,

$$
\int_{\Gamma} \frac{\partial}{\partial n_{x}}\left(\frac{1}{|x-y|}\right) d \gamma_{x}=\Omega_{y}
$$

where $\Omega_{y}$ is the solid angle sustained by the surface $\Gamma$ at the point $y$. Therefore,

$$
\frac{\partial}{\partial n_{y}}\left\{\int_{\Gamma} \frac{\partial}{\partial n_{x}}\left(\frac{1}{|x-y|}\right) d \gamma_{x}\right\}=0
$$

and

$$
C=\frac{1}{8 \pi} \int_{\Gamma} q(y) q^{\prime}(y) \frac{\partial}{\partial n_{y}}\left\{\int_{\Gamma} \frac{\partial}{\partial n_{x}}\left(\frac{1}{|x-y|}\right) d \gamma_{x}\right\} d \gamma_{y}=0
$$

Moreover, since

$$
\forall w \in K,\left\langle\frac{\partial w}{\partial n}, 1\right\rangle=0
$$

we have

$$
D=\frac{1}{8 \pi} \int_{\Gamma} \frac{\partial}{\partial n_{y}}\left\{\int_{\Gamma} q(x) q^{\prime}(x) \frac{\partial}{\partial n_{x}}\left(\frac{1}{|x-y|}\right) d \gamma_{x}\right\} d \gamma_{y}=0
$$

Finally, we arrive at

$$
a\left(q, q^{\prime}\right)=A+B+C+D
$$


Then, we can write

$$
a\left(q, q^{\prime}\right)=\frac{1}{8 \pi} \int_{\Gamma} \frac{\partial}{\partial n_{y}}\left\{\int_{\Gamma}(q(y)-q(x))\left(q^{\prime}(y)-q^{\prime}(x)\right) \frac{\partial}{\partial n_{x}}\left(\frac{1}{|x-y|}\right) d \gamma_{x}\right\} d \gamma_{y}
$$

provided that we do not forget that the differentiation with respect to $n_{y}$ does not concern $q(y)$ and $q^{\prime}(y)$. This can be seen in the expressions of $A, B$ and $C$.

It becomes possible then to interchange the order of integration and differentiation. This leads us to

$$
a\left(q, q^{\prime}\right)=\frac{1}{8 \pi} \int_{\Gamma} \int_{\Gamma}(q(y)-q(x))\left(q^{\prime}(y)-q^{\prime}(x)\right) \frac{\partial^{2}}{\partial n_{x} \partial n_{y}}\left(\frac{1}{|x-y|}\right) d \gamma_{x} d \gamma_{y}
$$

Remark. The same arguments can be used for the exterior Neumann problem in $\mathbf{R}^{2}$. This time, the space $W_{0}^{1}\left(\Omega^{c}\right)$ becomes

$$
W_{0}^{1}\left(\Omega^{c}\right)=\left\{\begin{array}{l|l}
v \in D^{\prime}\left(\Omega^{c}\right) \mid \frac{v}{r \log r} \in L^{2}\left(\Omega^{c}\right), D v \in L^{2}\left(\Omega^{c}\right)
\end{array}\right\} .
$$

Problem $\left(P_{1}\right)$ becomes

$$
\left\{\begin{array}{l}
\text { Find } u \in W_{0}^{1}\left(\Omega^{c}\right) / \mathbf{R} \text { such that } \\
\Delta u=0 \text { in } \Omega^{c} \\
\partial u / \partial n=g \in H_{0}^{-1 / 2}(\Gamma)
\end{array}\right.
$$

for now, $\|\operatorname{grad} u\|_{L^{2}\left(\Omega^{c}\right)}$ is a norm only on $W_{0}^{1}\left(\Omega^{c}\right) / \mathbf{R}$ equivalent to the quotient norm [4].

Then, we can immediately find a harmonic extension of $u$ inside $\Omega$. Thus, Problem $(P)$ can be written

$$
\left\{\begin{array}{l}
\text { Find } u \in\left(H^{1}(\Omega) / \mathbf{R}\right) \times\left(W_{0}^{1}\left(\Omega^{c}\right) / \mathbf{R}\right) \text { such that } \\
\Delta u=0 \text { in } \Omega \text { and } \Omega^{c} \\
\partial u / \partial n=g \in H_{0}^{-1 / 2}(\Gamma)
\end{array}\right.
$$

Concerning Problem $\left(P^{\prime}\right)$, the only change will be the definition of

$$
W^{1}\left(\Delta ; \Omega^{c}\right)=\left\{v \mid v \in W_{0}^{1}\left(\Omega^{c}\right), r \log r \Delta v \in L^{2}\left(\Omega^{c}\right)\right\}
$$

Finally, for regular $q$ and $q^{\prime}$, the solution of Problem $\left(P^{\prime}\right)$ can be written as

$$
u(y)=\frac{1}{2 \pi} \int_{\Gamma} q(x) \frac{\partial}{\partial n_{x}} \log |x-y| d \gamma_{x}+C,
$$

where $C$ is an arbitrary constant; the bilinear form $a$ becomes

$$
a\left(q, q^{\prime}\right)=-\frac{1}{4 \pi} \int_{\Gamma} \int_{\Gamma}(q(x)-q(y))\left(q^{\prime}(x)-q^{\prime}(y)\right) \frac{\partial^{2}}{\partial n_{x} \partial n_{y}} \log |x-y| d \gamma_{x} d \gamma_{y} .
$$


II. Approximation. The problem is to approximate the solution $q$ of

$$
\left\{\begin{array}{l}
\text { Find } q \in H^{1 / 2}(\Gamma) / \mathbf{R} \text { such that } \\
a\left(q, q^{\prime}\right)=\left\langle g, q^{\prime}\right\rangle, \forall q^{\prime} \in H^{1 / 2}(\Gamma) / \mathbf{R}
\end{array}\right.
$$

1. Construction of an Approximate Surface $\Gamma_{h}[14]$. Let $\left\{O_{i}\right\}$ be a family of $p$ bounded open sets of $\mathbf{R}^{3}$, covering $\Gamma$ and such that, for each $i$, there exists a $C^{\infty}$ mapping

$$
\mathrm{O}_{i} \stackrel{\theta_{i}}{\rightarrow} Q=\left\{y\left|y=\left\{y^{\prime}, y_{3}\right\},\right| y^{\prime} \mid<1,-1<y_{3}<1\right\}
$$

Let us further assume that $\theta_{i}$ has a $C^{\infty}$ inverse mapping $Q \stackrel{\theta_{i}^{-1}}{\longrightarrow} O_{i}$ and that $\theta_{i}$ is a mapping of $\mathcal{O}_{i} \cap \Omega, O_{i} \cap \Omega^{c}, O_{i} \cap \Gamma$, onto, respectively,

$Q_{-}=\left\{y \in Q \mid y_{3}<0\right\}, \quad Q_{+}=\left\{y \in Q \mid y_{3}>0\right\}, \quad Q_{0}=\left\{y \in Q \mid y_{3}=0\right\}$.

We shall assume that the usual compatibility relations [10] hold between the $\theta_{i}$.

In what follows, we shall write $\varphi_{i}$ for $\theta_{i}^{-1}$ considered as a mapping of $Q_{0}$ onto $O_{i} \cap \Gamma$

To define $\Gamma_{h}$, let us assume that we know a partition of $\Gamma$ into $p$ closed parts $\Gamma_{i}$ such that

$$
\Gamma_{i} \subset \mathcal{O}_{i}, \quad \bigcup_{i=1}^{P} \Gamma_{i}=\Gamma, \quad \Gamma_{i} \cap \Gamma_{j} \text { is a curve of } \Gamma \text { (or empty), when } i \neq j .
$$

Let us denote by $D_{i}$ the image of $\Gamma_{i}$ by $\theta_{i}$.

Let $\Sigma_{h}$ be a set of nodes on $\Gamma$, and let $\sigma_{i h}$ be the image of $\Sigma_{h} \cap \Gamma_{i}$ by $\theta_{i}$. Now, we build on $\sigma_{i h}$ a triangulation $T_{i n}$ of $D_{i}$. Then, to each element $T$ of each triangulation $T_{i h}$, we append a $C^{0}$ Lagrange finite element with an interpolation space $G$ such that $P_{k} \subset G$, where $P_{k}$ is the space of polynomials of degree $k$ or less.

Let $\varphi_{i h}$ be the mapping the restriction of which, on each element $T$ of $T_{i h}$, is the $G$ interpolate $F_{T}$ of $\varphi_{i}$. Then, $\Gamma_{h}$ is the surface defined by the mappings $\varphi_{i h}$.

2. Approximation $n_{h}$ of the Normal $n$ to $\Gamma$. We will use, as an approximation to $n$, the $G$ interpolate $n_{h}$ of $n$. As we shall see later, this is consistent with the approximation chosen for $\Gamma$.

3. Construction of an Approximation $V_{h}$ of $H^{1 / 2}(\Gamma)$. To each element $T$ of the triangulation

$$
T_{h}=\bigcup_{i=1}^{P} T_{i h}
$$

we associate a functional vector space $P$, such that $P_{m} \subset P$.

Then, we define $V_{h}$ as the space of the images of the elements of $P$ on every curved element of $\Gamma_{h}$ by the mapping $F_{T}$, i.e.,

$$
V_{h}=\left\{q_{h} \in C^{0}\left(\Gamma_{h}\right)\left|q_{h}\right|_{T}=p \circ F_{T}^{-1}, \forall T \in T_{h}, \forall p \in P\right\} .
$$

We want the elements of $V_{h}$ to be continuous on $\Gamma_{h}$, in order to have the inclusion of $V_{h}$ in $H^{1 / 2}\left(\Gamma_{h}\right)$. 
4. The Approximate Problem. The kernel of Problem (Q) is

$$
\frac{\partial^{2}}{\partial n_{x} \partial n_{y}}\left(\frac{1}{|x-y|}\right)=\frac{\left(n_{x}, n_{y}\right)}{|x-y|^{3}}-3 \frac{\left(x-y, n_{x}\right)\left(x-y, n_{y}\right)}{|x-y|^{5}} .
$$

Let us approximate this kernel by

$$
\frac{\left(n_{h x}, n_{h y}\right)}{|x-y|^{3}}-3 \frac{\left(x-y, n_{h x}\right)\left(x-y, n_{h y}\right)}{|x-y|^{5}},
$$

where $x, y$ belong to $\Gamma_{h}$, and $n_{h x}, n_{h y}$ are the approximations of $n_{x}$ and $n_{y}$, defined in II-2.

We shall write

$$
\frac{\partial^{2}}{\partial n_{h x} \partial n_{h y}}\left(\frac{1}{|x-y|}\right)
$$

for this kernel. Then, we approximate Problem ( 2 ) by

$$
\left(Q_{h}\right)\left\{\begin{array}{l}
\text { Find } q_{h} \in V_{h} / \mathbf{R} \text { such that } \\
-\frac{1}{8 \pi} \int_{\Gamma_{h}} \int_{\Gamma_{h}}\left(q_{h}(x)-q_{h}(y)\right)\left(q_{h}^{\prime}(x)-q_{h}^{\prime}(y)\right) \frac{\partial^{2}}{\partial n_{h x} \partial n_{h y}}\left(\frac{1}{|x-y|}\right) d \gamma_{h x} d \gamma_{h y} \\
=\int_{\Gamma_{h}} g_{h}(y) q_{h}^{\prime}(y) d \gamma_{h y}, \forall q_{h}^{\prime} \in V_{h} / \mathbf{R},
\end{array}\right.
$$

where $g_{h}$ is an approximation of $g$ defined on $\Gamma_{h}$ and satisfying $\left\langle g_{h}, 1\right\rangle=0$.

We shall write Problem $\left(Q_{h}\right)$ more concisely as

$$
\left\{\begin{array}{l}
\text { Find } q_{h} \in V_{h} / \mathbf{R} \text { such that } \\
a_{h}\left(q_{h}, q_{h}^{\prime}\right)=\left\langle g_{h}, q_{h}^{\prime}\right\rangle{ }_{H_{0}^{-1 / 2}\left(\Gamma_{h}\right) \times\left(H^{1 / 2}\left(\Gamma_{h}\right) / \mathbf{R}\right)}, \forall q_{h}^{\prime} \in V_{h} / \mathbf{R} .
\end{array}\right.
$$

According to Theorem I-2, that we apply now to Problem $\left(Q_{h}\right)$, this problem would have one and only one solution if $n_{h}$ was normal to $\Gamma_{h}$.

Unfortunately, this is not the case because, in order to get an optimal order of convergence, we have chosen another $n_{h}$. Thus, the existence of a unique solution to Problem $\left(Q_{h}\right)$ will be a consequence of the uniform coercivity of the bilinear forms $a_{h}$. This uniform coercivity will appear during the error study of the following section.

5. Error Estimates. To compare $q$ and $q_{h}$, we have to define a mapping of $\Gamma_{h}$ onto $\Gamma$. For the same reasons as those of [14], we have to use $\psi$ defined by

-for $x$ belonging to $\Gamma_{h}, \psi(x)$ is the orthogonal projection of $x$ onto $\Gamma$, i.e., $\psi(x)$ is the foot of the normal to $\Gamma$ passing through $x$.

For $\Gamma_{h}$ sufficiently close to $\Gamma$, i.e., for $h$ sufficiently small, $\psi$ is regular and bijective [14].

Then, we have the following estimates,

TheOREM II-1. Let $q$ and $q_{h}$ be the solutions, respectively, of Problems (2) and $\left(Q_{h}\right)$. Then, if $q$ belongs to $H^{m+1}(\Gamma)$, we have 


$$
\begin{aligned}
& \left\|q-q_{h} \circ \psi^{-1}\right\|_{H^{1 / 2}(\Gamma) / \mathrm{R}} \\
& \quad \leqslant C\left\{\left\|g-\hat{g}_{h}\right\|_{H_{0}^{-1 / 2}(\Gamma)}+h^{m+1 / 2} \|_{\left.q\left\|_{H^{m+1}}\right\|_{(\Gamma) / \mathrm{R}}+h^{k+1}\|q\|_{H^{1 / 2}(\Gamma) / \mathrm{R}}\right\}}\right.
\end{aligned}
$$

and

$$
\begin{aligned}
\left\|q-q_{h} \circ \psi^{-1}\right\|_{L^{2}(\Gamma) / \mathrm{R}} \leqslant C\left\{\sqrt{h}\left\|g-\hat{g}_{h}\right\|_{H_{0}^{-1 / 2}(\Gamma)}+\left\|g-\hat{g}_{h}\right\|_{H_{0}^{-1}(\Gamma)}\right. \\
\left.+h^{m+1}\|q\|_{H^{m+1}(\Gamma) / \mathrm{R}}+h^{k+1}\|q\|_{H^{1 / 2}(\Gamma) / \mathrm{R}}\right\},
\end{aligned}
$$

where

$$
\hat{g}_{h}(x)=\left(g_{h} \circ \psi^{-1}(x) J\left(\psi^{-1}(x)\right)\right) .
$$

Proof. Let $\widetilde{V}_{h}$ be the subspace of $H^{1 / 2}(\Gamma)$, the image of $V_{h}$ by the mapping $\psi^{-1}$ i.e.,

$$
\widetilde{V}_{h}=\left\{\tilde{v_{h}} \mid \tilde{v_{h}}=v_{h} \circ \psi^{-1}, \forall v_{h} \in V_{h}\right\}
$$

Let us consider Problem $\left(Q_{h}\right)$ on $\Gamma$. Defining $J$ as Jacobian mapping, we obtain: find $\widetilde{q}_{h} \in \widetilde{V}_{h} / \mathbf{R}$ such that

$$
\begin{gathered}
-\frac{1}{8 \pi} \int_{\Gamma} \int_{\Gamma}\left(\tilde{q}_{h}(x)-\tilde{q}_{h}(y)\right)\left(\widetilde{q}_{h}^{\prime}(x)-\tilde{q}_{h}^{\prime}(y)\right) \frac{\partial^{2}}{\partial n_{h x} \partial n_{h y}}\left(\frac{1}{\left|\psi^{-1}(x)-\psi^{-1}(y)\right|}\right) \\
\cdot J\left(\psi^{-1}(x)\right) J\left(\psi^{-1}(y)\right) d \gamma_{x} d \gamma_{y} \\
=\int_{\Gamma} \tilde{g}_{h}(y) \widetilde{q}_{h}^{\prime}(y) J\left(\psi^{-1}(y)\right) d \gamma_{y}, \quad \forall \widetilde{q}_{h}^{\prime} \in \widetilde{V}_{h} / \mathbf{R} .
\end{gathered}
$$

Define $\hat{g}_{h}=\widetilde{g}_{h} J\left(\psi^{-1}\right)$, then, $\left\langle\hat{g}_{h}, 1\right\rangle=0$; and we have the following estimate $\left\|q-\tilde{q}_{h}\right\|_{H^{1 / 2}(\Gamma) / R}$

$$
\begin{aligned}
& \leqslant C\left\{\left\|g-\hat{g}_{h}\right\|_{H_{0}^{-1 / 2}(\Gamma)}\right. \\
& \left.\quad+\inf _{\tilde{q}_{h}^{\prime} \in \tilde{V}_{h} / \mathrm{R}}\left[\left\|q-\tilde{q}_{h}^{\prime}\right\|_{H^{1 / 2}(\Gamma) / \mathrm{R}}+\sup _{w_{h} \in \tilde{V}_{h} / \mathrm{R}} \frac{\left|a\left(\tilde{q}_{h}^{\prime}, w_{h}\right)-a_{h}\left(q_{h}^{\prime}, w_{h}\right)\right|}{\left\|w_{h}\right\|_{H^{1 / 2}}(\Gamma) / \mathrm{R}}\right]\right\} .
\end{aligned}
$$

Let us choose $\widetilde{q}_{h}^{\prime}=\Pi_{h} q$, where $\Pi_{h}$ is the $\widetilde{V}_{h}$ interpolation operator. We know then [1] that

$$
\left\|q-\Pi_{h} q\right\|_{L^{2}(\Gamma)} \leqslant C h^{m+1}\|q\|_{H^{m+1}(\Gamma)},
$$

and

$$
\left\|q-\Pi_{h} q\right\|_{H^{1}(\Gamma)} \leqslant C h^{m}\|q\|_{H^{m+1}(\Gamma)} ;
$$

hence, by interpolating between $L^{2}(\Gamma)$ and $H^{1}(\Gamma)$, 


$$
\left\|q-\Pi_{h} q\right\|_{H^{1 / 2}(\Gamma)} \leqslant C h^{m+1 / 2} \|_{q H^{m+1} \|_{(\Gamma)}}
$$

and since

$$
(q+C)-\Pi_{h}(q+C)=q-\Pi_{h} q, \quad \forall C \in \mathbf{R},
$$

we have

$$
\left\|q-\Pi_{h} q\right\|_{H^{1 / 2}(\Gamma) / \mathbf{R}} \leqslant C h^{m+1 / 2} \|_{H^{m+1}(\Gamma) / \mathbf{R}} .
$$

It remains to estimate the error due to the change of bilinear form. For this, we shall need

LEMma II-1. The quantity

$$
b(q)=\left\{\int_{\Gamma} \int_{\Gamma} \frac{(q(x)-q(y))^{2}}{|x-y|^{3}} d \gamma_{x} d \gamma_{y}\right\}^{1 / 2}
$$

is a norm on $H^{1 / 2}(\Gamma) / \mathbf{R}$ equivalent to the usual norm.

Proof of Lemma II-1. According to Theorems I-2 and I-3, $a(q)$ defined by

$$
a(q)=\left\{\int_{\Gamma} \int_{\Gamma}(q(x)-q(y))^{2} \frac{\partial^{2}}{\partial n_{x} \partial n_{y}}\left(\frac{1}{|x-y|}\right) d \gamma_{x} d \gamma_{y}\right\}^{1 / 2}
$$

is a norm on $H^{1 / 2}(\Gamma) / \mathbf{R}$ equivalent to the usual one. But

$$
\frac{\partial^{2}}{\partial n_{x} \partial n_{y}}\left(\frac{1}{|x-y|}\right)=\frac{\left(n_{x}, n_{y}\right)}{|x-y|^{3}}-3 \frac{\left(x-y, n_{x}\right)\left(x-y, n_{y}\right)}{|x-y|^{5}}
$$

so that

$$
\left|\frac{\partial^{2}}{\partial n_{x} \partial n_{y}}\left(\frac{1}{|x-y|}\right)\right| \leqslant \frac{4}{|x-y|^{3}} \text { and } a(q) \leqslant 4 b(q) .
$$

On the other hand,

$$
\frac{\partial^{2}}{\partial n_{x} \partial n_{y}}\left(\frac{1}{|x-y|}\right)=\frac{1}{|x-y|^{3}}+\frac{f(x, y)}{|x-y|},
$$

where $f(x, y)$ is bounded in a neighborhood of $x=y$, so that

$$
b^{2}(q) \leqslant a^{2}(q)+\int_{\Gamma} \int_{\Gamma}(q(x)-q(y))^{2} \frac{f(x, y)}{|x-y|} d \gamma_{x} d \gamma_{y}
$$

and since

$$
\int_{\Gamma} \frac{|f(x, y)|}{|x-y|} d \gamma_{x}
$$

is a bounded function of $y$, by developing $(q(x)-q(y))^{2}$, we obtain 


$$
b^{2}(q) \leqslant a^{2}(q)+C\left\|_{q}\right\|_{L^{2}(\Gamma)}^{2}
$$

Moreover, $b(q)$ equals 0 when $q$ is a constant, so that Lemma II-1 is proved.

We shall use three other lemmas proved in [5]. Let us give them.

Lемма II-2. Let $h$ be the greatest diameter of the elements $T$ of $T_{h}$, then

$$
\begin{array}{r}
\max _{i=1, P} \sup _{x \in S_{i}}\left|\Phi_{i}(x)-\Phi_{i h}(x)\right| \leqslant C h^{k+1} \max _{i=1, P} \sup _{x \in S_{i}}\left\|D^{k+1} \Phi_{i}(x)\right\|, \\
\max _{i=1, P} \sup _{x \in S_{i}}\left|D^{l} \Phi_{i}(x)-D^{l} \Phi_{i h}(x)\right| \leqslant C h^{k+1-l} \max _{i=1, P} \sup _{x \in S_{i}}\left\|D^{k+1} \Phi_{i}(x)\right\|,
\end{array}
$$

with $1 \leqslant|l| \leqslant k+1$.

LEMma II-3. For each triangle $T$ of $T_{h}$, the mapping $\psi \circ F_{T}$ is bounded as also its derivatives up to the order $k+1 . D\left(\psi \circ F_{T}\right)$ is a linear mapping of rank 2 , having a bounded inverse when considered as a mapping of $\mathbf{R}^{2}$ onto the tangent plane to $\Gamma$. Moreover,

$$
\begin{gathered}
\sup _{x \in T}\left|\psi \circ F_{T}(x)-F_{T}(x)\right| \leqslant C h^{k+1} \sup _{x \in T}\left|D^{k+1} \Phi_{i}(x)\right|, \\
\sup _{x \in T}\left|D\left(\psi \circ F_{T}\right)(x)-D F_{T}(x)\right| \leqslant C h^{k} \sup _{x \in T}\left|D^{k+1} \Phi_{i}(x)\right| .
\end{gathered}
$$

Lemma II-4. Let $T$ and $T^{\prime}$ be two triangles of $T_{h}$. Then,

$$
\begin{gathered}
\sup _{x \in T}\left|J\left(F_{T}\right)(x)-J\left(\psi \circ F_{T}\right)(x)\right| \leqslant C h^{k+1} \sup _{x \in T}\left|D^{k+1} \Phi_{i}(x)\right|, \\
C\left|F_{T}(x)-F_{T^{\prime}}(y)\right| \leqslant\left|\psi \circ F_{T}(x)-\psi \circ F_{T^{\prime}}(y)\right| \leqslant C\left|F_{T}(x)-F_{T^{\prime}}(y)\right|, \\
|| F_{T}(x)-\left.F_{T^{\prime}}(y)\right|^{2}-\left|\psi \circ F_{T}(x)-\psi \circ F_{T^{\prime}}(y)\right|^{2}\left|\leqslant C h^{k+1}\right| F_{T}(x)-\left.F_{T^{\prime}}(y)\right|^{2} .
\end{gathered}
$$

The first inequality of this last lemma shows why we are interested in the mapping $\psi$. It enables us to obtain an error on the Jacobian mapping of order $k+1$, instead of $k$.

We are now in a position to resume the proof of Theorem II-1.

The error due to the change of bilinear form can be split up into three parts

-the error due to the change of Jacobian mapping;

-the error due to the change of normal;

-the error due to the substitution of $\psi^{-1}(x)$ to $x$.

Using Lemmas II-1 and II-4, we can see immediately that the first error is bounded by

$$
C h^{k+1}\|q\|_{H^{1 / 2}(\Gamma) / \mathrm{R}} .
$$

As for the second and third errors, we have to estimate 


$$
\begin{gathered}
\frac{\partial^{2}}{\partial n_{x} \partial n_{y}}\left(\frac{1}{|x-y|}\right)-\frac{\partial^{2}}{\partial n_{h x} \partial n_{h y}}\left(\frac{1}{\left|\psi^{-1}(x)-\psi^{-1}(y)\right|}\right) \\
=\frac{\left(n_{x}, n_{y}\right)}{|x-y|^{3}}-\frac{\left(n_{h x}, n_{h y}\right)}{\left|\psi^{-1}(x)-\psi^{-1}(y)\right|^{3}}-3 \frac{\left(x-y, n_{x}\right)\left(x-y, n_{y}\right)}{|x-y|^{5}} \\
+3 \frac{\left(\psi^{-1}(x)-\psi^{-1}(y), n_{h x}\right)\left(\psi^{-1}(x)-\psi^{-1}(y), n_{h y}\right)}{\left|\psi^{-1}(x)-\psi^{-1}(y)\right|^{5}} .
\end{gathered}
$$

The error on the normal gives two kinds of terms

$$
\frac{\left(n_{x}-n_{h x}, n_{y}\right)}{|x-y|^{3}} \text { and } \frac{\left(x-y, n_{x}-n_{h x}\right)\left(x-y, n_{y}\right)}{|x-y|^{5}} \text {. }
$$

We shall examine the first one. Let us recall that $n_{h}$ is the $G$-interpolate of $n$, so that $\left|n_{x}-n_{h x}\right| \leqslant C h^{k+1}$, both terms are bounded by $C h^{k+1} /|x-y|^{3}$, and the error due to the change of normal can eventually be estimated by $C h^{k+1}\|q\|_{H^{1 / 2}(\Gamma) / R}$.

Let us now examine the error due to the substitution of $\psi^{-1}(x)$ to $x$. There appear three kinds of terms.

$$
\begin{gathered}
\frac{1}{|x-y|^{3}}-\frac{1}{\left|\psi^{-1}(x)-\psi^{-1}(y)\right|^{3}}, \frac{1}{|x-y|^{5}}-\frac{1}{\left|\psi^{-1}(x)-\psi^{-1}(y)\right|^{5}} \\
\left(x-y, n_{x}\right)-\left(\psi^{-1}(x)-\psi^{-1}(y), n_{x}\right) .
\end{gathered}
$$

For the first term, we put

$$
A=|x-y| ; \quad B=\left|\psi^{-1}(x)-\psi^{-1}(y)\right| .
$$

Then,

$$
\begin{aligned}
\frac{1}{A^{3}}-\frac{1}{B^{3}} & =\frac{B^{3}-A^{3}}{A^{3} B^{3}}=\frac{(B-A)\left(B^{2}+A B+A^{2}\right)}{A^{3} B^{3}} \\
& =\frac{\left(B^{2}-A^{2}\right)\left(B^{2}+A B+A^{2}\right)}{A^{3} B^{3}(A+B)}
\end{aligned}
$$

According to Lemma II-4, this last term is bounded above by $C h^{k+1} /|x-y|^{3}$. An analogous argument gives the same bound for the second term.

The third term can be bounded in two ways.

On the one hand, we have

$$
\left|x-y-\psi^{-1}(x)+\psi^{-1}(y)\right| \leqslant\left|x-\psi^{-1}(x)\right|+\left|y-\psi^{-1}(y)\right| \leqslant C h^{k+1},
$$

and, on the other hand, we also have

$$
\left|x-y-\psi^{-1}(x)+\psi^{-1}(y)\right|=\left|\left(I-\psi^{-1}\right)(x)-\left(I-\psi^{-1}\right)(y)\right| \leqslant C h^{k}|x-y| .
$$

Thus, terms like 


$$
\begin{aligned}
\frac{\left(x-y-\psi^{-1}(x)+\psi^{-1}(y), n_{x}\right)\left(x-y, n_{h x}\right)}{|x-y|^{5}} & \\
= & \frac{\left(x-y-\psi^{-1}(x)+\psi^{-1}(y), n_{x}\right)\left(x-y, n_{x}\right)}{|x-y|^{5}} \\
& +\frac{\left(x-y-\psi^{-1}(x)+\psi^{-1}(y), n_{x}\right)\left(x-y, n_{h x}-n_{x}\right)}{|x-y|^{5}}
\end{aligned}
$$

can be bounded above by

$$
\frac{C h^{k+1}|x-y|^{2}}{|x-y|^{5}}+\frac{C h^{k}|x-y| C h^{k+1}|x-y|}{|x-y|^{5}} \leqslant C \frac{h^{k+1}}{|x-y|^{3}}
$$

and the error due to the substitution of $\psi^{-1}(x)$ to $x$ is bounded by $C h^{k+1}\|q\|_{H^{1 / 2}(\Gamma) / R}$, which completes the estimation of the error in $H^{1 / 2}(\Gamma) / R$.

The error in $L^{2}(\Gamma) / \mathbf{R}$ will be given by the following theorem.

THEOREM II-2. Let $s \leqslant m$. We have the following error estimate

$$
\begin{aligned}
& \left\|q-\tilde{q}_{h}\right\|_{H^{-s}(\Gamma) / \mathrm{R}} \leqslant C\left\{h^{s+1 / 2}\left\|g-\hat{g}_{h}\right\|_{H_{0}^{-1 / 2}(\Gamma)}+\left\|g-\hat{g}_{h}\right\|_{H_{0}^{-s-1}(\Gamma)}\right. \\
& \left.+h^{m+s+1}\|q\|_{H^{m+1}(\Gamma) / \mathrm{R}}+h^{k+1} \|_{q \|_{H^{1 / 2}(\Gamma) / \mathrm{R}}}\right\} \text {. }
\end{aligned}
$$

Proof. We shall need

LEMMA II-5. Let $q$ be the solution of

$$
\left\{\begin{array}{l}
\text { Find } q \in H^{1 / 2}(\Gamma) / \mathbf{R} \text { such that } \\
a\left(q, q^{\prime}\right)=\left\langle g, q^{\prime}\right\rangle_{H_{0}^{-1 / 2}(\Gamma) \times\left(H^{1 / 2}(\Gamma) / \mathbf{R}\right)}, \forall q^{\prime} \in H^{1 / 2}(\Gamma) / \mathbf{R},
\end{array}\right.
$$

then,

$$
\forall s \geqslant 0, \quad g \in H_{0}^{s}(\Gamma) \Rightarrow q \in H^{s+1}(\Gamma) / \mathbf{R} \text { and }\|q\|_{H^{s+1}(\Gamma) / \mathbf{R}} \leqslant C\|g\|_{H_{0}^{s}(\Gamma)} .
$$

Proof of Lemma II-5. Let $u$ be the solution of

$$
\left\{\begin{array}{l}
\text { Find } u \in\left(H^{1}(\Omega) / \mathbf{R}\right) \times W_{0}^{1}\left(\Omega^{c}\right) \text { such that } \\
\Delta u=0 \text { in } \Omega \text { and } \Omega^{c} \\
\partial u / \partial n=g \in H_{0}^{-1 / 2}(\Gamma) .
\end{array}\right.
$$

Then, the classical regularity theorems tell us that

$$
\left.g \in H_{0}^{s}(\Gamma) \Rightarrow u\right|_{\text {int }} \in H^{s+3 / 2}(\Omega) / R \text { and }\left.u\right|_{\text {ext }} \in H_{\text {loc }}^{s+3 / 2}\left(\Omega^{c}\right) \text {, }
$$

hence $q=[u] \in H^{s+1}(\Gamma) / \mathbf{R}$. The closed graph theorem applied to the mapping $g$ $\rightarrow q$ ends the proof of this lemma.

Proof of Theorem II-2. We shall use a classical duality argument. We have

$$
\left\|q-\tilde{q}_{h}\right\|_{H^{-s}(\Gamma) / \mathrm{R}}=\sup _{g \in H_{0}^{s}(\Gamma)} \frac{\left|\left(g, q-\tilde{q}_{h}\right)\right|}{\|g\|_{H_{0}^{s}(\Gamma)}}
$$


but

$$
\left(g, q-\tilde{q}_{h}\right)=a\left(q-\tilde{q}_{h}, J g\right)
$$

where $J$ is the mapping $\partial u / \partial n \rightarrow[u]$, defined according to Proposition I-2.

Then, Lemma II-5 tells us that $\|J g\|_{H^{s+1}(\Gamma) / R} \leqslant C\left\|_{g}\right\|_{H_{0}^{s}(\Gamma)}$, so that

$$
\left\|q-\tilde{q}_{h}\right\|_{H^{-s}(\Gamma) / \mathbf{R}} \leqslant C \sup _{g \in H_{0}^{s}(\Gamma)} \frac{\left|a\left(q-\tilde{q}_{h}, J g\right)\right|}{\|J g\|_{H^{s+1}}(\Gamma) / \mathbf{R}} .
$$

Now, we have

$$
a\left(q-\tilde{q}_{h}, J g\right)=a\left(q-\tilde{q}_{h}, J g-\Pi_{h} J g\right)+a\left(q-\tilde{q}_{h}, \Pi_{h} J g\right)
$$

where $\Pi_{h}$ is the $\widetilde{V}_{h}$ interpolation operator. Therefore,

$$
\left\|J g-\Pi_{h} J g\right\|_{H^{1 / 2}(\Gamma) / \mathrm{R}} \leqslant C h^{s+1 / 2}\|J g\|_{H^{s+1}(\Gamma) / \mathrm{R}},
$$

so that

$$
\sup _{g \in H_{0}^{s}(\Gamma)} \frac{\left|a\left(q-\tilde{q}_{h}, J g-\Pi_{h} J g\right)\right|}{\|J g\|_{H^{s+1}(\Gamma) / \mathbf{R}}} \leqslant C h^{s+1 / 2}\left\|q-\widetilde{q}_{h}\right\|_{H^{1 / 2}(\Gamma) / \mathbf{R}} .
$$

It remains to estimate the second term

$$
\begin{aligned}
& a\left(q-\tilde{q}_{h}, \Pi_{h} J g\right)=\left(g, \Pi_{h} J g\right)-a\left(\tilde{q}_{h}, \Pi_{h} J g\right)=\left(g, \Pi_{h} J g\right)-\left(\hat{g}_{h}, \Pi_{h} J g\right) \\
&-\frac{1}{8 \pi} \int_{\Gamma} \int_{\Gamma}\left(\widetilde{q}_{h}(x)-\widetilde{q}_{h}(y)\right)\left(\Pi_{h} J g(x)-\Pi_{h} J g(y)\right) \\
& \cdot\left\{\frac{\partial^{2}}{\partial n_{x} \partial n_{y}}\left(\frac{1}{|x-y|}\right)-\frac{\partial^{2}}{\partial n_{h x} \partial n_{h y}}\left(\frac{1}{\left|\psi^{-1}(x)-\psi^{-1}(y)\right|}\right)\right. \\
&\left.\cdot J\left(\psi^{-1}(x)\right) J\left(\psi^{-1}(y)\right)\right\} d \gamma_{x} d \gamma_{y} .
\end{aligned}
$$

The last term can be studied in the same way as for the proof of Theorem II-1. This study leads to the estimate $C h^{k+1}\|q\|_{H^{1 / 2}(\Gamma) / R}\|J g\|_{H^{1 / 2}(\Gamma) / R}$. As for the difference $\left(g, \Pi_{h} J g\right)-\left(\hat{g}_{h}, \Pi_{h} J g\right)$, it can be bounded above by $\left\|g-\hat{g}_{h}\right\|_{H_{0}^{-s-1}(\Gamma)}$. $\|J g\|_{H^{s+1}(\Gamma) / \mathrm{R}}$, which gives

$$
\sup _{g \in H_{0}^{s}(\Gamma)} \frac{\left|a\left(q-\tilde{q}_{h}, \Pi_{h} J g\right)\right|}{\|J g\|_{H^{s+1}(\Gamma) / \mathrm{R}}} \leqslant C h^{k+1}\left\|_{q \|_{H^{1 / 2}(\Gamma) / \mathrm{R}}}+\right\| g-\hat{g}_{h} \|_{H_{0}^{-s-1}(\Gamma)},
$$

and ends the proof of Theorem II-2.

The $L^{2}$ estimate announced in Theorem II-1 can be obtained by choosing $s=0$.

Finally, we must not forget that our first problem was to find the solution of Problem $(P)$. In that respect, the following theorem is the more interesting. 
Theorem II-3. Let $u$ be the solution of Problem $(P)$ satisfying $\int_{\Gamma} q(x) d \gamma_{x}=0$, and let $u_{h}$ be defined by

$$
u_{h}(y)=-\frac{1}{4 \pi} \int_{\Gamma_{h}} q_{h}(x) \frac{\partial}{\partial n_{h x}}\left(\frac{1}{|x-y|}\right) d \gamma_{h x},
$$

where $q_{h}$ is the solution of Problem $\left(2_{h}\right)$ satisfying $\int_{\Gamma_{h}} q_{h}(x) d \gamma_{h x}=0$. Let us assume that $\exists \delta>0: d(y, \Gamma) \geqslant \delta$, then, for sufficiently small $h$, e.g. for $h$ such that

$$
\sup _{z \in \Gamma_{h}} d(z, \Gamma) \leqslant \frac{d(y, \Gamma)}{2}
$$

we have the following error estimate

$$
\begin{aligned}
\left|u(y)-u_{h}(y)\right| \leqslant \frac{C}{e(y, \Gamma)}\left\{h^{m+1 / 2}\left\|g-\hat{g}_{h}\right\|_{H_{0}^{-1 / 2}(\Gamma)}+\left\|g-\hat{g}_{h}\right\|_{H_{0}^{-m-1}(\Gamma)}\right. \\
\left.+h^{2 m+1}\|q\|_{H^{m+1}(\Gamma) / \mathrm{R}}+h^{k+1} \|_{H_{H} \|_{(\Gamma) / \mathrm{R}}}\right\},
\end{aligned}
$$

where

$$
\frac{1}{e(y, \Gamma)}=\sum_{n=0}^{m} \frac{1}{d^{2+n}(y, \Gamma)}+ \begin{cases}1 & \text { when } y \in \Omega \\ 0 & \text { when } y \in \Omega^{c}\end{cases}
$$

Proof. We have

$$
\begin{aligned}
u(y)-u_{h}(y)= & -\frac{1}{4 \pi} \int_{\Gamma}\left[q(x)-\tilde{q}_{h}(x) J\left(\psi^{-1}(x)\right)\right] \frac{\partial}{\partial n_{x}}\left(\frac{1}{|x-y|}\right) d \gamma_{x} \\
& -\frac{1}{4 \pi} \int_{\Gamma} \tilde{q}_{h}(x) J\left(\psi^{-1}(x)\right)\left\{\frac{\partial}{\partial n_{x}}\left(\frac{1}{|x-y|}\right)-\frac{\partial}{\partial n_{h x}}\left(\frac{1}{\left|\psi^{-1}(x)-y\right|}\right)\right\} d \gamma_{x} .
\end{aligned}
$$

Now, since

$$
\int_{\Gamma}\left[q(x)-\tilde{q}_{h}(x) J\left(\psi^{-1}(x)\right)\right] d \gamma_{x}=\int_{\Gamma} q d \gamma-\int_{\Gamma_{h}} q_{h} d \gamma_{h}=0
$$

we have

$$
\begin{aligned}
\int_{\Gamma}[q(x)- & \left.\tilde{q}_{h}(x) J\left(\psi^{-1}(x)\right)\right] \frac{\partial}{\partial n_{x}}\left(\frac{1}{|x-y|}\right) d \gamma_{x} \\
& =\int_{\Gamma}\left[q(x)-\tilde{q}_{h}(x) J\left(\psi^{-1}(x)\right)\right]\left[\frac{\partial}{\partial n_{x}}\left(\frac{1}{|x-y|}\right)+\frac{4 \pi}{\operatorname{mes}(\Gamma)}\right] d \gamma_{x}
\end{aligned}
$$

On the other hand, we have

$$
\int_{\Gamma} \frac{\partial}{\partial n_{x}}\left(\frac{1}{|x-y|}\right) d \gamma_{x}= \begin{cases}-4 \pi & \text { when } y \in \Omega, \\ 0 & \text { when } y \in \Omega^{c}\end{cases}
$$

so that 


$$
\begin{aligned}
& \left|\int_{\Gamma}\left[q(x)-\tilde{q}_{h}(x) J\left(\psi^{-1}(x)\right)\right] \frac{\partial}{\partial n_{x}}\left(\frac{1}{|x-y|}\right) d \gamma_{x}\right| \\
& \leqslant\left\|q-\tilde{q}_{h} J\left(\psi^{-1}\right)\right\|_{H^{-m}(\Gamma) / \mathbf{R}} \times\left\{\begin{array}{l}
\left\|\frac{\partial}{\partial n_{x}}\left(\frac{1}{|x-y|}\right)+\frac{4 \pi}{\operatorname{mes}(\Gamma)}\right\|_{H_{0}^{m}(\Gamma)} \text { when } y \in \Omega, \\
\left\|\frac{\partial}{\partial n_{x}}\left(\frac{1}{|x-y|}\right)\right\|_{H_{0}^{m}(\Gamma)}, \quad \text { when } y \in \Omega^{c} .
\end{array}\right.
\end{aligned}
$$

Now,

$$
\left\|q-\tilde{q}_{h} J\left(\psi^{-1}\right)\right\|_{H^{-m}(\Gamma) / \mathbf{R}} \leqslant\left\|q-\tilde{q}_{h}\right\|_{H^{-m}(\Gamma) / \mathbf{R}}+\left\|\tilde{q}_{h}\left(1-J\left(\psi^{-1}\right)\right)\right\|_{H^{-m}}(\Gamma) / \mathbf{R}
$$

and

$$
\left\|\frac{\partial}{\partial n_{x}}\left(\frac{1}{|x-y|}\right)\right\|_{H^{m}(\Gamma)}=\left\|\frac{\left(n_{x}, x-y\right)}{|x-y|^{3}}\right\|_{H^{m}(\Gamma)} \leqslant C \sum_{n=0}^{m} \frac{1}{d^{2+n}(y, \Gamma)}
$$

so that we can proceed to the second term

$$
\begin{aligned}
\mid \int_{\Gamma} \tilde{q}_{h}(x) J\left(\psi^{-1}(x)\right) & \left\{\frac{\partial}{\partial n_{x}}\left(\frac{1}{|x-y|}\right)-\frac{\partial}{\partial n_{h x}}\left(\frac{1}{\left|\psi^{-1}(x)-y\right|}\right)\right\} d \gamma_{x} \mid \\
\leqslant\left\|\tilde{q}_{h} J\left(\psi^{-1}\right)\right\|_{L^{2}(\Gamma)} & \left\|\frac{\partial}{\partial n_{x}}\left(\frac{1}{|x-y|}\right)-\frac{\partial}{\partial n_{h x}}\left(\frac{1}{\left|\psi^{-1}(x)-y\right|}\right)\right\|_{L^{2}(\Gamma)} ;
\end{aligned}
$$

but since

$$
\int_{\Gamma} \tilde{q}_{h} J\left(\psi^{-1}\right) d \gamma=\int_{\Gamma_{h}} q_{h} d \gamma_{h}=0
$$

we have

$$
\left\|\tilde{q}_{h} J\left(\psi^{-1}\right)\right\|_{L^{2}(\Gamma)}=\left\|q_{h}\right\|_{L^{2}\left(\Gamma_{h}\right) / \mathrm{R}} \leqslant C \|_{L^{2}(\Gamma) / \mathrm{R}} .
$$

On the other hand,

$$
\begin{aligned}
&\left\|\frac{\partial}{\partial n_{x}}\left(\frac{1}{|x-y|}\right)-\frac{\partial}{\partial n_{h x}}\left(\frac{1}{\left|\psi^{-1}(x)-y\right|}\right)\right\|_{L^{2}(\Gamma)} \\
&=\left\|\frac{\left(n_{x}, x-y\right)}{|x-y|^{3}}-\frac{\left(n_{h x}, \psi^{-1}(x)-y\right)}{\left|\psi^{-1}(x)-y\right|^{3}}\right\|_{L^{2}(\Gamma)} \\
& \leqslant\left\|\frac{\left(n_{x}, x-y\right)-\left(n_{h x}, \psi^{-1}(x)-y\right)}{|x-y|^{3}}\right\|_{L^{2}(\Gamma)} \\
&+\left\|\left(n_{h x}, \psi^{-1}(x)-y\right)\left\{\frac{1}{|x-y|^{3}}-\frac{1}{\left|\psi^{-1}(x)-y\right|^{3}}\right\}\right\|_{L^{2}(\Gamma)} \leqslant \frac{C h^{k+1}}{d^{3}(y, \Gamma)}
\end{aligned}
$$

and this ends the proof of Theorem II-3. 
The same type of estimate can be obtained for the derivatives. More specifically, we have

THEOREM II4. Under the hypotheses of Theorem II-3, we have the following estimate

$$
\begin{aligned}
\left|\partial^{\alpha} u(y)-\partial^{\alpha} u_{h}(y)\right| \leqslant \frac{C}{e_{\alpha}(y, \Gamma)}\left\{h^{m+1 / 2}\left\|g-\hat{g}_{h}\right\|_{H_{0}^{-1 / 2}(\Gamma)}+\left\|g-\hat{g}_{h}\right\|_{H_{0}^{-m-1}(\Gamma)}\right. \\
\left.+h^{2 m+1}\|q\|_{H^{m+1}(\Gamma) / \mathrm{R}}+h^{k+1}\|q\|_{H^{1 / 2}(\Gamma) / \mathrm{R}}\right\},
\end{aligned}
$$

where

$$
\frac{1}{e_{\alpha}(y, \Gamma)}=\sum_{n=0}^{m} \frac{1}{d^{2+|\alpha|+n}(y, \Gamma)}+ \begin{cases}1, & \text { when } y \in \Omega, \\ 0, & \text { when } y \in \Omega^{c}\end{cases}
$$

Proof. The proof is the same as that of Theorem II-3.

Remark. For the two-dimensional case, the same techniques give the same results.

III. A Few Numerical Remarks.

1. Condition Number for the Matrix $a_{h}$. For $h$ small enough, we have

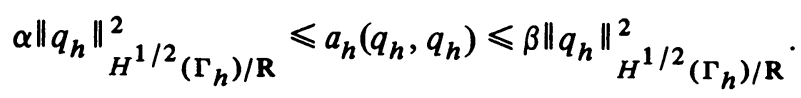

If we choose $q_{h}$ such that $\int_{\Gamma_{h}} q_{h} d \gamma_{h}=0$, we get, according to Lemma II-1,

$$
\alpha\left\|q_{h}\right\|_{H^{1 / 2}\left(\Gamma_{h}\right)}^{2} \leqslant a_{h}\left(q_{h}, q_{h}\right) \leqslant \beta\left\|q_{h}\right\|_{H^{1 / 2}\left(\Gamma_{h}\right)}^{2} .
$$

Now, since $q_{h}$ belongs to $V_{h}$, we have [15]

$$
\left\|q_{h}\right\|_{H^{1 / 2}\left(\Gamma_{h}\right)} \leqslant \frac{C}{\sqrt{h}}\left\|q_{h}\right\|_{L^{2}\left(\Gamma_{h}\right)},
$$

so that

$$
\alpha\left\|q_{h}\right\|_{L^{2}\left(\Gamma_{h}\right)}^{2} \leqslant a_{h}\left(q_{h}, q_{h}\right) \leqslant \frac{\beta}{h}\left\|q_{h}\right\|_{L}^{2}\left(\Gamma_{h}\right),
$$

which shows that the eigenvalues $\lambda$ of the matrix $a_{h}$ are bounded above and below by $\alpha \leqslant \lambda \leqslant \beta / h$, so that the condition number of $a_{h}$ is of $O\left(h^{-1}\right)$ order.

2. Computation of the Elements of $a_{h}$ Near the Diagonal. To compute these elements, we have to integrate a function with a singularity of order $1 /|x-y|$. When $k$ and $m$ are not too high, we can use primitives. In that case, the following theorem is of some interest.

THEOREM III-1. Let us assume that, when $|x-y| \leqslant C h$ we define in the kernel of $a_{h}$, i.e. in

$$
\frac{\left(n_{h x}, n_{h y}\right)}{|x-y|^{3}}-3 \frac{\left(x-y, n_{h x}\right)\left(x-y, n_{h y}\right)}{|x-y|^{5}}
$$


$n_{h x}$ and $n_{h y}$ as the normals to $\Gamma_{h}$ in $x$ and $y$, respectively (instead of using interpolates of order $k$ of $n_{x}$ and $n_{y}$ ).

Then, the estimate of Theorem II-1 remains valid.

Proof. We only have to estimate

$$
\frac{\left(n_{x}-n_{h x}, n_{y}\right)}{|x-y|^{3}} \text { and } \frac{\left(x-y, n_{x}-n_{h x}\right)\left(x-y, n_{y}\right)}{|x-y|^{5}},
$$

when $|x-y| \leqslant C h$. Let us examine the first term. We have $n_{y}=n_{x}+O(x-y)$, so that

$$
\left(n_{x}-n_{h x}, n_{y}\right)=\left(n_{x}-n_{h x}, n_{x}\right)+\left(n_{x}-n_{h x}, O(x-y)\right)
$$

But

$$
\left|\left(n_{x}-n_{h x}, O(x-y)\right)\right| \leqslant C h^{k} . C h=C h^{k+1},
$$

so that it remains to estimate $\left(n_{x}-n_{h x}, n_{x}\right)$.

Let $\left(x_{1}, x_{2}\right)$ be the two components of $x$. We get

$$
n_{x}=\frac{\partial\left(\psi \circ \varphi_{i h}\right) / \partial x_{1} \wedge \partial\left(\psi \circ \varphi_{i h}\right) / \partial x_{2}}{\left|\partial\left(\psi \circ \varphi_{i h}\right) / \partial x_{1} \wedge \partial\left(\psi \circ \varphi_{i h}\right) / \partial x_{2}\right|}=\frac{1}{J\left(\psi \circ \varphi_{i h}\right)}\left(\frac{\partial\left(\psi \circ \varphi_{i h}\right)}{\partial x_{1}} \wedge \frac{\partial\left(\psi \circ \varphi_{i h}\right)}{\partial x_{2}}\right),
$$

and

$$
n_{h x}=\frac{1}{J\left(\varphi_{i h}\right)}\left(\frac{\partial \varphi_{i h}}{\partial x_{1}} \wedge \frac{\partial \varphi_{i h}}{\partial x_{2}}\right) .
$$

According to Lemma II -4 , the error on $J$ is of order $k+1$. We have now to estimate terms like

$$
R=\left(\frac{\partial\left(\psi \circ \varphi_{i h}\right)}{\partial x_{1}} \wedge\left(\frac{\partial\left(\psi \circ \varphi_{i h}\right)}{\partial x_{2}}-\frac{\partial \varphi_{i h}}{\partial x_{2}}\right), \frac{\partial\left(\psi \circ \varphi_{i h}\right)}{\partial x_{1}} \wedge \frac{\partial\left(\psi \circ \varphi_{i h}\right)}{\partial x_{2}}\right)
$$

Such terms have been shown to be of order $k+1$ in [14, p. 67] by using the fact that $\psi$ is the orthogonal projection onto $\Gamma$, so that we obtain

$$
\left(n_{x}-n_{h x}, n_{y}\right) \leqslant C h^{k+1} .
$$

For the second term, it is much easier. We have

$$
\frac{\left|\left(x-y, n_{x}-n_{h x}\right)\left(x-y, n_{y}\right)\right|}{|x-y|^{5}} \leqslant \frac{C h^{k}|x-y||x-y|^{2}}{|x-y|^{5}} \leqslant \frac{C h^{k+1}}{|x-y|^{3}},
$$

since $\left(x-y, n_{y}\right)=O\left(|x-y|^{2}\right)$.

However, the involved primitives are difficult to compute, so that we are led to the use of numerical integration. The kernel being singular near the diagonal, we use extrapolation to the limit techniques [12] which give excellent results.

Finally, for more details on the numerical aspects and results of the method described in this paper, we refer to [5].

Conclusion. We have shown how to use a double layer potential to solve the Neumann problem without introducing Cauchy type integrals. 
Thanks to the variational formulation thus obtained, we have been able to prove error estimates. We have seen that the error on the jump of the solution through $\Gamma$ is optimal when $k=m$, whereas the error on the solution, far enough from $\Gamma$, is optimal when $k=2 m$. As for the condition number of the matrix, it is of order $O\left(h^{-1}\right)$.

These results can be compared with those obtained by J. C. Nedelec [14] for the Dirichlet problem, by the use of a single layer potential. There, the error on the jump of $\partial u / \partial n$ through $\Gamma$ was optimal for $k=m+1$, and the condition number of the matrix was of the same order $O\left(h^{-1}\right)$. However, the smallest eigenvalue of the matrix was only of order $O(h)$, so that the coercivity of $a_{h}$ was very sensitive to numerical errors. This last fact appeared in the numerical experiments of M. Djaoua [3] .

Centre de Mathématiques Appliquées

Ecole Polytechnique

91128 Palaiseau Cedex, France

1. P. G. CIARLET, The Finite Element Method for Elliptic Problems, North-Holland, Amsterdam, 1977.

2. P. G. CIARLET \& P. A. RAVIART, "General Lagrange and Hermite interpolation in $\mathbf{R}^{n}$ with applications to finite element methods," Arch. Rational Mech. Anal., v. 46, 1972, pp. 177199.

3. M. DJAOUA, Méthode d'Éléments Finis pour la Résolution d'un problème extérieur dans $\mathbf{R}^{3}$, Rapport Interne du Centre de Mathématiques Appliquées de l'Ecole Polytechnique, no. 3, 1975.

4. J. GIROIRE, Formulation Variationnelle par Équations Intégrales de Problèmes aux Limites Extérieurs, Rapport Interne du Centre de Mathématiques Appliquées de l'Ecole Polytechnique, no. 6, 1976 .

5. J. GIROIRE, Mise en Oeuvre Numérique de la Résolution par Potentiel de Double Couche du Probleme de Neumann Extérieur, Rapport Interne du Centre de Mathématiques Appliquées de l'Ecole Polytechnique. (To appear.)

6. B. HANOUZET, "Espaces de Sobolev avec poids. Application au problème de Dirichlet dans un demi-espace," Rend. Sem. Mat. Univ. Padova, v. 46, 1971, pp. 247-272.

7. G. C. HSIAO \& W. WENDLAND, "A finite element method for some integral equations of the first kind," J. Math. Anal. Appl., v. 58, 1977, pp. 449-481.

8. M. N. LEROUX, "Equations intégrales pour le problème du potentiel électrique dans le plan," C. R. Acad. Sci. Paris, Sér. Math. A, v. 178, 1974, pp. 541-544.

9. J. L. LIONS, Problèmes aux Limites dans les Equations aux Dérivées Partielles, Presses de l'Université de Montréal, 1965.

10. J. L. LIONS \& E. MAGENES, Problèmes aux Limites Non Homogènes et Applications, Dunod, Paris, 1968.

11. J. N. LYNESS, "An error functional expansion for $N$-dimensional quadrature with an integrand function singular at a point," Math. Comp., v. 30, 1976, pp. 1-23.

12. J. N. LYNESS, “Applications of extrapolation techniques to multidimensional quadrature of some integrand functions with a singularity," J. Computational Phys., v. 20, 1976, pp.346-364.

13. S. G. MIKHLIN, Mathematical Physics, An Advanced Course, North-Holland, Amsterdam, 1970.

14. J. C. NEDELEC, "Curved finite element methods for the solution of singular integral equations on surfaces in $\mathrm{R}^{3}$," Comput. Methods Appl. Mech. Engrg., v. 8, 1976, pp. 61-80.

15. J. C. NEDELEC \& J. PLANCHARD, "Une méthode variationnelle d'éléments finis pour la résolution numérique d'un problème extérieur dans $\mathbf{R}^{3}$," Rev. Française Informat. Recherche Operationnelle Ser. Rouge 3, v. 7, 1973, pp. 105-129.

16. P. P. ZABREYKO, et al., Integral Equations, A Reference Text, Noordhoff, Leyden, 1975.

17. O. C. ZIEN KIEWICZ, The Finite Element Method in Engineering Science, McGrawHill, London, 1971. 